report with inconsistent information on laterality itself. It correctly extracts $83 \%$ of 465 reports for assessment for right breast and $92 \%$ for assessment for left breast. Unstable gold standard impedes performance of the NLP algorithm for extracting parenchymal enhancement and clinical indications. There is no gold standard to show NLP performance for comparison exams yet. Conclusions: This NLP algorithm holds promise for rapid, accurate extraction of information from free-text breast MRI reports. Manual review will be faster and more accurate due to the pre-filling of the abstraction form. Keywords: Natural language processing; Breast MRI report

doi:10.3121/cmr.2014.1250.ps1-15

PS1-16:

Effect of Insurance Plan Choice on Utilization in Kaiser Permanente Colorado

Nikki Carroll ${ }^{1}$; Laurie Crounse ${ }^{1}$; Jeffrey Holzman ${ }^{1}$; Carsie Nyirenda ${ }^{1}$

\section{${ }^{1}$ Kaiser Permanente Colorado}

Background/Aims: There has been a shift in recent years from traditional HMO-type insurance products to high deductible or co-insurance plans. Kaiser Permanente Colorado (KPCO) has also added new service areas to the region that include Southern and Northern Colorado. In addition, the impending Affordable Care Act will be implemented in 2014 which may bring new and different members to KPCO. There is a need to describe sociodemographic and clinical characteristics by plan type in order to understand how members within these different plan types utilize KPCO resources and what data are available and identifiable within our source data systems. These answers will enable us to establish appropriate inclusion and exclusion criteria for future research studies. Methods: All members with at least 1 day of membership from January, 2000 through July 2013 were identified. Membership within product lines and all associated utilization was based on calendar year. Patient characteristics, ambulatory visits, inpatient admissions, emergency department admissions, lab tests, tumors, pharmacy utilization and variations in therapy for certain diagnoses were obtained from the Virtual Data Warehouse (VDW). Socio-demographic characteristics, including gender, race, and age, were described by percentages. Clinical characteristics and variation in utilization were calculated on a per-person per-month measure and were compared for each region/insurance product/year combination. Results: Demographics and utilization varied across all product lines and years. An increase in the number of different products offered was observed over time. Members in the HMO plan type were more likely to be younger, less educated and use more resources than members in other products. Some data were not easily identifiable in certain plan types. Conclusions: These results indicate that demographic and clinical patterns do vary between plan types. These patterns need to be taken into account when creating cohorts in future research projects.

Keywords: Utilization; Insurance plans

doi:10.3121/cmr.2014.1250.ps1-16

PS1-17:

Organization-wide Collaboration to Convert Binary Clinical Notes to Research-ready Text

Scott Halgrim ${ }^{1}$; Shane Reeh ${ }^{2}$;Roger Kelem²; Roger Wong ${ }^{2}$; Aruna Kamineni ${ }^{1}$; Virginia Immanuel ${ }^{1}$; Christopher Pattillo ${ }^{2}$; Roy Pardee ${ }^{1}$; David Carrell ${ }^{1}$; Carolyn Rutter ${ }^{1}$

\section{${ }^{1}$ Group Health Research Institute; ${ }^{2}$ Group Health}

Background/Aims: Clinical text is an integral part of research at Group Health Research Institute (GHRI), supporting ongoing projects and new research proposals. Our clinical notes are stored in a single, full-text-indexed table that is populated nightly with all new notes in Group Health's Electronic Medical Record (EMR). These notes include those generated internally and those received from partner organizations via electronic interfaces. Recently one of our partners changed its interface from sending formatted text to sending binary PDF files. While this had little effect on clinical users, it made a significant amount of text data inaccessible for computerized processing. This text was crucial to ongoing research, so we needed to find a way to preserve access. Methods: A diversely-skilled team of technologists collaborated to solve the problem with the following process. When the nightly HL7 file arrives at Group Health, it is copied to a research server and the message ID is stored with the appropriate encounter in the EMR. A scheduled Python script then extracts the message ID and binary PDF from each HL7 message. It converts the base64-encoded information into a PDF file and names it with the message ID for linking with the encounter. Finally, the script converts the PDF file into a text file using an open source library. The PDF and text files are archived, as is the nightly HL7 message. Results: From June to October 2013, this process has converted and archived over 30,000 notes from our partner that otherwise would not have been available for research. The files are also available to Group Health's claims auditing department, enabling Group Health to see a monetary return on the effort. Conclusions: Thanks to the open source community and strong connections between informatics management at Group Health Cooperative and GHRI, we were able to quickly salvage critical data for research when an externally-driven change to that information occurred. Before the end of 2013 we expect to integrate these converted text notes with our clinical text storage.

Keywords: Natural language processing; Data interfaces doi:10.3121/cmr.2014.1250.ps1-17

PS1-18:

Do Patients Use Online Messaging to Substitute for Office Visits and Phone Calls? Results from a Natural Experiment

Laura Panattoni $^{1}$; Sean McClellan ${ }^{1}$; Ming Tai-Seale ${ }^{1}$

${ }^{1}$ Palo Alto Medical Foundation Research Institute

Background/Aims: We evaluated the effect of online messaging between patients and providers on other healthcare utilization. Messaging may increase the administrative burden on providers but may also substitute for utilization such as calls or visits. To identify the effect of messaging, we exploited a natural experiment, which occurred in $3 / 2011$, when a large multispecialty practice in California lowered the price of messaging from $\$ 60 /$ year to free for patients and initiated incentives for doctors ( $\$ 3 /$ thread), resulting in increased rates of patients messaging. Methods: We conducted a longitudinal study of 65,332 active primary care patients from 3/2009$3 / 2012$. All patients in the study had activated their online portal before $3 / 2011$ and none had paid to use messaging previously; 38,438 of these patients initiated a message following 3/2011. Messaging was measured by the number of threads initiated by patients annually. Outcome measures included annual rates of office visits and phone calls to primary and specialty care. The effects of messaging were identified through difference-indifferences analyses using generalized least squares, comparing the utilization of those initiating any messages with those not messaging, before and after messaging became free. Controls included patient fixed effects along with patient age, insurance type, Charlson score, continuity of care, and relevant characteristics of their primary care provider. Results: Comparing the rates of service use before and after 3/2011, between messaging and nonmessaging users, we found no difference in the annual number of primary care phone calls $(0.002$ per patient-year, $P=0.394)$, and a small increase in primary care office visits $(0.05$ per patient-year, $P<0.001)$ for each thread initiated. For specialty care, we found an increase in 0.16 phone calls $(P$ $<0.001)$ and $0.21(P<0.001)$ office visits per patient-year for each thread initiated. Conclusions: Among patients who were already using the online portal, use of secure messaging was associated with little difference in primary care utilization, but with a moderate increase in specialty utilization. Although current analyses controlled for patient and provider characteristics, patient self-selection may still threaten validity; we will address this through propensity score stratification in future analyses.

Keywords: Online messaging; Healthcare utilization doi:10.3121/cmr.2014.1250.ps1-18

\section{Health Services Research/Health Policy/Costs}

B2-1:

Estimation of Standardized Hospital Costs from Claims Data that Reflect Resource Requirements for Care

John Schousboe'; Misti Paudel²; Brent Taylor²; Lih-Wen Mah³ Beth Virnig²; Kristine Ensrud ${ }^{2}$; Bryan Dowd ${ }^{2}$ 